\title{
Rolle der Elektronik
}

Liebe Leserin, lieber Leser,

der Automobilbranche stehen dramatische Umwälzungen bevor. Bis 2025 soll sich Prognosen zufolge weltweit jedes vierte Fahrzeug elektrisch fortbewegen. Zusätzlich stellen Zukunftstechnologien wie autonomes und vernetztes Fahren sowie völlig neue datengetriebene Geschäftsmodelle höchste Anforderungen an Hersteller und Zulieferer. Für Sie als Besucher der electronica stellt sich daher die Frage, welche Rolle die angesprochenen Themen auf der Weltleitmesse und Konferenz der Elektronik einnehmen werden.

Der treibende Faktor im Bereich Automotive heißt Elektronik. Deshalb erhalten Sie auf der electronica einen umfassenden Überblick zu Trends, Produkten, Technologien und Märkten der Automobilindustrie. Informieren Sie sich bei den Ausstellern in den Messehallen, im Automotive Forum sowie in der electronica Automotive Conference (eAC).

Nutzen Sie die Möglichkeit, die electronica Automotive Conference mit ihren hochkarätigen Vorträgen am Montag, 12. November, zu besuchen. Am Tag vor dem offiziellen Messebeginn stehen dort wesentliche Technologietrends und Strategien der weltweiten Automobilindustrie im Mittelpunkt. Einen der Schwerpunkte neben emissionsfreiem und autonomem Fahren bis Level 5 bildet das zukunftsweisende Thema Last Mile als Teil des Mobilitätsmix. Besonders freue ich mich auf die Keynotes zur Eröffnung des Konferenzprogramms von Rainer Müller-Finkeldei, Director of Mechatronic Development bei Daimler, sowie Jürgen Bortolazzi, Director of Driver Assistance Systems bei Porsche.

Während der Messe empfehle ich Ihnen das electronica Automotive Forum in der Halle B4. Fachvorträge und Podiumsdiskussionen an allen vier Messetagen ermöglichen es Ihnen, Ihr Fachwissen zu erweitern und neue Kontakte zu knüpfen. Diskutieren Sie zum Beispiel mit Elektronikentwicklern, Systemdesignern oder Supply-Chain-Managern über aktuelle Markt- und Technologiethemen.

In Halle B4 finden Sie zudem Aussteller und Produkte aus dem Bereich Automobil. Unter anderem präsentiert das japanische Technologieunternehmen Asahi Kasei (Stand B4.516) eine Europapremiere. Das Concept Car AKXY besteht unter anderem aus Lithium-Ionen-Batterieapplikationen, $\mathrm{CO}_{2}$-Sensoren sowie Hochleistungskunststoffen.

Neben den bereits erwähnten Informationen bietet die electronica 2018 selbstverständlich noch weitaus mehr Highlights. Ich lade Sie daher herzlich ein, sich selbst ein Bild vor Ort zu machen und vom 13. bis zum 16. November die Weltleitmesse und Konferenz der Elektronik in München zu besuchen.

Ich wünsche Ihnen gute und gewinnbringende Gespräche.

\section{Angela Marten}

Projektleiterin electronica,

Messe München

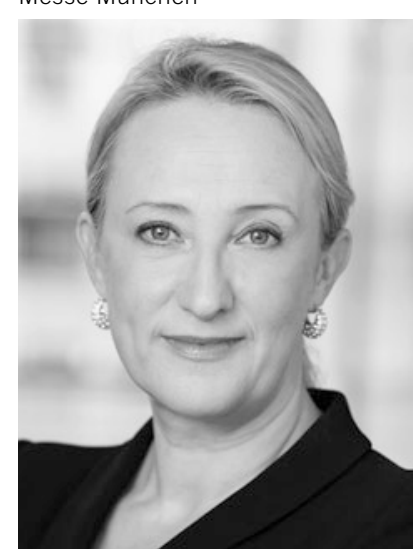

